\title{
Association of mean platelet volume and red blood cell distribution width with coronary collateral development in stable coronary artery disease
}

\author{
Isa Sincer ${ }^{1}$, Yilmaz Gunes ${ }^{1}$, Asli Kurtar Mansiroglư ${ }^{1}$, Mehmet Cosgun $^{1}$, Gulali Aktas $^{2}$ \\ 'Department of Cardiology, Abant Izzet Baysal University Hospital, Bolu, Turkey \\ ${ }^{2}$ Department of Internal Medicine, Abant Izzet Baysal University Hospital, Bolu, Turkey
}

Adv Interv Cardiol 2018; 14, 3 (53): 263-269

DOI: https://doi.org/10.5114/aic.2018.78329

\begin{abstract}
A bstract
Introduction: The prognostic value of hematological indices in cardiovascular diseases and the association between these parameters and cardiovascular conditions have been established in the literature.

Aim: In this study, we aimed to investigate the relation of mean platelet volume (MPV), MPV to platelet ratio (MPR) and red cell distribution width (RDW) with degree of coronary collateral development (CCD) in stable coronary artery disease (CAD) subjects with established critical coronary artery stenosis.

Material and methods: A total of 306 patients with stable angina pectoris undergoing coronary arteriography were enrolled and divided on the basis of the development of CCD into two groups: a group with adequate CCD $(n=214)$ and a group with impaired CCD $(n=92)$. Routine complete blood count and biochemical parameters were measured before coronary arteriography.

Results: The MPV and MPR levels were significantly higher in the inadequate CCD group $(10.5 \pm 1.8 \mathrm{fl} \mathrm{vs.} 8.7 \pm 1.9 \mathrm{fl}, p<0.001$ and $0.06 \pm 0.08$ vs. $0.05 \pm 0.07, p=0.036$ ). Patients with inadequate CCD had significantly higher RDW levels compared to patients with adequate CCD $(15.5 \pm 1.7 \%$ vs. $15.0 \pm 1.9 \%, p=0.01)$. MPV and RDW were significantly associated with Rentrop collateral grading $(r=-0.523, p<0.001$ and $r=-0.239, p<0.001$, respectively), whereas the association with MPR was not significant. An MPV value greater than $9.95 \mathrm{fl}$, determined with ROC curve analysis, had $71 \%$ sensitivity and $70 \%$ specificity in predicting inadequate CCD. An RDW greater than $14.3 \%$ has $71 \%$ sensitivity and 53\% specificity in selecting patients with adequate CCD.

Conclusions: The present study suggests that MPV and MPR may be associated with the degree of collateral development in chronic stable CAD. However, the negative association of RDW with inadequate CCD, in combination with previous contradictory reports, raises a doubt about the possible value of RDW in stable CAD. Although these parameters may be affected by various conditions, a high MPV may lead clinicians to suspect possible inadequate collateral development in stable CAD patients.
\end{abstract}

Key words: mean platelet volume, coronary collateral development, coronary artery disease, red cell distribution width, mean platelet volume to platelet ratio.

Su m m a ry

In this study, we aimed to investigate the relation of mean platelet volume (MPV), MPV to platelet ratio (MPR) and red cell distribution width (RDW) with degree of coronary collateral development (CCD) in stable coronary artery disease (CAD) subjects with established critical coronary artery stenosis. The present study suggests that MPV and MPR may be associated with the degree of collateral development in chronic stable CAD. However, the negative association of RDW with inadequate CCD, in combination with previous contradictory reports.

\section{Introduction}

Coronary collateral circulation develops as an alternative source of blood supply when the main blood supply ceases as a result of critical or total occlusion of the main coronary artery [1]. The amount of coronary collateral development (CCD) differs among patients even within those having the same degree of stenosis [2]. Lack of collateral vessels in some patients despite the presence

\section{Corresponding author:}

Isa Sincer MD, Department of Cardiology, Abant Izzet Baysal University Hospital, Golkoy, 14200, Bolu, Turkey, phone: +90 3742534656, fax: +90 3742534615, e-mail: isasincer@yahoo.com

Received: 5.03.2018, accepted: 6.05 .2018 
of coronary obstruction suggests the contribution of additional factors to the collateral development. Although several factors, such as severity and duration of myocardial ischemia, diabetes mellitus (DM), hypertension (HT), dyslipidemia and smoking, have been reported to affect coronary angiogenesis and collateral growth [3], the underlying mechanism for variation in collateral circulation development is still unclear.

Previous studies have shown the association between coronary artery disease (CAD) and some hematological parameters including elevated red cell distribution width (RDW) and platelet indices (CAD) [4-10]. Platelet activity plays a crucial role in progression of atherosclerosis $[2,11]$. Platelet size has been linked to its activity more accurately than platelet count [12, 13]. Furthermore, a high ratio of mean platelet volume (MPV) to platelet count (MPR) is considered to be associated with various conditions including myocardial infarction, anemia, and hepatocellular carcinoma [12-15]. Sahin et al. demonstrated that inadequate CCD was independently associated with RDW, but not with any other hematological parameters including MPV in patients with stable CAD [2]. However, Ege et al. found MPV to be an independent predictor of impaired CCD [8].

\section{Aim}

In this study, we aimed to investigate the relation of MPV, MPR and RDW with degree of CCD in stable CAD patients documented with coronary angiography.

\section{Material and methods}

The patients who had undergone coronary angiography in our institution between June 2014 and December 2017 were consecutively evaluated for the presence of coronary collateral development. The patients had stable angina and/or ischemic findings on electrocardiography or stress tests. Patients who had at least $95 \%$ luminal narrowing in at least one major epicardial coronary artery were included. Clinical and laboratory findings were obtained from review of the patients' files. Hypertension was defined as blood pressure $>140 / 90 \mathrm{~mm} \mathrm{Hg}$ or receiving antihypertensive medication. Diabetes mellitus was defined as having a fasting glucose level $>126 \mathrm{mg} / \mathrm{dl}$ or receiving antidiabetic medication. Presence of total cholesterol > $200 \mathrm{mg} / \mathrm{dl}$ or triglyceride > $150 \mathrm{mg} / \mathrm{dl}$ was accepted as hyperlipidemia. Patients with a history of acute coronary syndrome within 6 months, history of coronary artery stenting or bypass operation, idiopathic dilated or hypertrophic cardiomyopathy, congestive heart failure, moderate to severe renal failure, severe hepatic dysfunction, atrial fibrillation, severe valvular disease, systemic inflammatory diseases (e.g. rheumatoid arthritis, lupus erythematosus), malignancy, history of blood transfusion within 3 months, recent infection (1 month), leukemia or thrombocytopenia, were excluded. The study was approved by the local ethics committee of the institution.

\section{Laboratory data}

Peripheral venous blood samples were drawn from stable CAD patients who were admitted for angiography or during regular follow-up checkups. Serum glucose, creatinine, total cholesterol, high-density lipoprotein cholesterol, and low-density lipoprotein cholesterol were measured using an automatic biochemical analyzer (Architect C8000, USA). Complete blood count and platelet volumes were determined using simultaneous optical and impedance measurements (Cell Dyn 3700; Abbott Diagnostics, Lake Forest, Illinois, USA). Platelet, RDW and MPV values of each patient were recorded. The MPV to platelet ratio (MPR) value was calculated by division of MPV by platelet count.

\section{Coronary angiography evaluation}

Coronary angiographies were performed through the radial or femoral artery. Patients having coronary artery stenosis of $\geq 95 \%$, by visual evaluation, were included. Coronary collateral circulation was graded according to the Rentrop classification [16]. Accordingly, grade 0 refers to a lack of filling in collateral vessels, grade 1 refers to filling in side branches via collateral channels without visualization of the epicardial artery, grade 2 refers to partial filling in the epicardial major coronary artery via collateral circulation, and grade 3 refers to complete filling in the epicardial major coronary artery. The coronary angiographies were evaluated by three interventional cardiologists who were blinded to the clinical and laboratory data of the patients. In the case of multiple coronary collateral circulation, the CCD with the highest Rentrop grade was used for analysis. The patients were as assigned to inadequate CCD (Rentrop grades $0-1$ ) and adequate CCD (Rentrop grades 2-3) groups.

\section{Statistical analysis}

Analyses were carried out using SPSS 15.0 for Windows (SPSS Inc, Chicago, Illinois, USA).

Quantitative variables are expressed as mean \pm standard deviation (SD), and qualitative variables as numbers and percentages. Differences between independent groups were assessed by Student's t-test for normally distributed quantitative variables and the Mann-Whitney $U$-test for variables without normal distribution and the $\chi^{2}$ test for qualitative variables. Pearson correlation analyses were used to assess the correlations of MVP, MPR, and RDW with Rentrop grade. Multivariate linear regression analysis was used to analyze the value of different baseline characteristics as independent predictors of inadequate CCD. Diagnostic ability of MVP, MPR and RDW to detect inadequate CCD was evaluated with receiver operating characteristic curves. To reveal the association 
of variables with adequate CCD we performed univariate analysis. A multivariate logistic regression model with the forward stepwise method was used for variables found significant in univariate analysis in order to determine the independent prognostic factors of adequate CCD. All results were considered statistically significant at the level of $p \leq 0.05$

\section{Results}

Three hundred six (220 males, 86 females) patients were enrolled in the study. Of the study population 214 (70\%) patients had inadequate CCD and 92 (30\%) had adequate CCD. Baseline patient demographics, including age, sex, and clinical risk factors, were similar between the groups, except that the current smoking rate was significantly lower in the poor CCD group (18\% vs. $67 \%$, $p=0.02$ ). Previous medications were also comparable between the two groups (Table I).

Most of the serum biochemistry of the subjects in inadequate and adequate CCD groups were not statistically different. However, compared to the inadequate CCD group total cholesterol $(p=0.01)$ and LDL cholesterol $(p=0.006)$ were significantly higher in the adequate CCD group (Table II). Angiographic characteristics of the patients according to the coronary collateral formation are summarized in Table III.

Although the platelet counts were not significantly different between the two groups, MPV and MPR levels were significantly higher in the inadequate CCD group $(10.5 \pm 1.8 \mathrm{fl}$ vs. $8.7 \pm 1.9 \mathrm{fl}, p<0.001$ and $0.06 \pm 0.08$ vs.
$0.05 \pm 0.07, p=0.036$ ). Patients with inadequate CCD had significantly higher RDW levels compared to patients with adequate CCD $(15.5 \pm 1.7 \%$ vs. $15 \pm 1.9 \%$, $p=0.01)$. The MPV and RDW were significantly related with Rentrop collateral grading $(r=-0.523, p<0.001$ and $r=0.239, p<0.001$, respectively), whereas the relation with MPR was not significant.

A MPV value greater than $9.95 \mathrm{fl}$, determined with ROC curve analysis, had $71 \%$ sensitivity and $70 \%$ specificity in indicating inadequate CCD (Figure 1). An RDW greater than $14.3 \%$ has $71 \%$ sensitivity and $53 \%$ specificity in selecting patients with adequate CCD (Figure 2). In the multivariate analysis forward stepwise model, smoking ( $p=0.002,95 \% \mathrm{Cl}$ for OR: $0.22-0.71)$, MPV $(p<0.001,95 \% \mathrm{Cl}$ for OR: $1.36-1.80)$ and age $(p=0.022$, 95\% Cl for OR: 0.942-0.995) were found to be statistically significantly different in the adequate CCD group compared to the inadequate CCD group.

\section{Discussion}

In the present study we found that MPV and MPR ratio were significantly higher and RDW was significantly lower in patients with stable angina pectoris having poor collateral development compared to the subjects with adequate collateral development. Poor collateral development was significantly related with higher MPV and lower RDW levels.

The presence of well-developed CCD has been associated with limitation of infarct size, protection or improvement of left ventricular function and mortality in patients with $C A D[17,18]$. Factors that affect the development

Table I. General characteristics of study groups

\begin{tabular}{|c|c|c|c|}
\hline Baseline characteristics & Inadequate CCD $(n=214)$ & Adequate CCD $(n=92)$ & $P$-value \\
\hline Age (mean \pm SD) [years] & $62.49 \pm 10.98$ & $64.51 \pm 9.55$ & 0.059 \\
\hline Body mass index $\left[\mathrm{kg} / \mathrm{m}^{2}\right]$ & $28.79 \pm 4.51$ & $28.58 \pm 4.69$ & 0.758 \\
\hline Male/female & $146 / 68$ & $74 / 18$ & 0.65 \\
\hline Hypertension & 127 (59\%) & $58(63 \%)$ & 0.544 \\
\hline Smoking & $39(18 \%)$ & $62(67 \%)$ & 0.022 \\
\hline Family history & $22(10 \%)$ & 7 (8\%) & 0.530 \\
\hline Diabetes mellitus & 87 (41\%) & $38(41 \%)$ & 0.994 \\
\hline Acetyl salicylate & $125(60 \%)$ & $60(65 \%)$ & 0.076 \\
\hline Clopidogrel & $30(14 \%)$ & $15(16 \%)$ & 0.605 \\
\hline Statin & $70(32 \%)$ & $28(30 \%)$ & 0.263 \\
\hline Calcium channel blocker & $27(16 \%)$ & $16(17 \%)$ & 0.334 \\
\hline ACE inhibitor & $55(26 \%)$ & $20(22 \%)$ & 0.460 \\
\hline ARB & $55(26 \%)$ & $22(24 \%)$ & 0.741 \\
\hline$\beta$-Blocker & $89(42 \%)$ & $34(37 \%)$ & 0.265 \\
\hline
\end{tabular}

$C C D$ - coronary collateral development, $A C E$ - angiotensin-converting enzyme, ARB - angiotensin receptor blocker, SD - standard deviation. 
of CCD include the duration and/or the severity of coronary artery stenosis, endothelial dysfunction, endogenous mediators, hypertension, diabetes mellitus, dyslipidemia, smoking, and drugs [19]. Endogenous mediators and neurohumoral factors may play a role in angiogenesis [20]. Furthermore, inflammatory cells, such as monocytes, lymphocytes and platelets, may affect CCD [11, 21-25].
Platelets play a crucial role in progression of atherosclerosis [11]. Platelet size, measured as MPV, is a marker of its function. Increased platelet volume is associated with increased platelet reactivity, increased platelet aggregation, and higher thrombotic potential [26]. It is known that cardiac dysfunction can cause platelet activation and increased MPV [27]. However, platelet

Table II. Laboratory data of study cohort

\begin{tabular}{|c|c|c|c|}
\hline Parameter & Inadequate CCD $(n=214)$ & Adequate CCD $(n=92)$ & $P$-value \\
\hline Creatinine $[\mathrm{mg} / \mathrm{dl}]$ & $1.11 \pm 1.0$ & $1.01 \pm 0.63$ & 0.656 \\
\hline Fasting plasma glucose $[\mathrm{mg} / \mathrm{dl}]$ & $131.69 \pm 69.60$ & $130.14 \pm 70.75$ & 0.199 \\
\hline LDL cholesterol [mg/dl] & $119.77 \pm 41.19$ & $109.12 \pm 48.35$ & 0.006 \\
\hline HDL cholesterol [mg/dl] & $41.57 \pm 9.76$ & $43.9 \pm 9.81$ & 0.072 \\
\hline Triglyceride [mg/dl] & $184.54 \pm 114.65$ & $183.02 \pm 145$ & 0.312 \\
\hline Total cholesterol [mg/dl] & $198.26 \pm 53.07$ & $185.26 \pm 51.07$ & 0.010 \\
\hline Hemoglobin $[\mathrm{g} / \mathrm{dl}]$ & $14.05 \pm 1.8$ & $13.8 \pm 1.48$ & 0.396 \\
\hline Hematocrit [g/dl] & $42.7 \pm 5.6$ & $42.01 \pm 4.36$ & 0.30 \\
\hline Red cell distribution width (\%) & $15.03 \pm 1.86$ & $15.54 \pm 1.69$ & 0.01 \\
\hline$M P V[f l]$ & $10.46 \pm 1.83$ & $8.76 \pm 1.89$ & $<0.001$ \\
\hline Platelet count [k/mm³] & $241.21 \pm 61.07$ & $232.51 \pm 65.29$ & 0.115 \\
\hline MPR (\%) & $0.059 \pm 0.075$ & $0.054 \pm 0.068$ & 0.036 \\
\hline PCT & $0.179 \pm 0.047$ & $0.184 \pm 0.046$ & 0.532 \\
\hline
\end{tabular}

$P C T$ - plateletcrit, MPR - MPV to platelet ratio, MPV - mean platelet volume.

Table III. Coronary angiographic laboratory findings of study population

\begin{tabular}{|c|c|c|c|}
\hline Parameter & $\begin{array}{l}\text { Inadequate CCD } \\
(n=214)\end{array}$ & $\begin{array}{c}\text { Adequate CCD } \\
(n=92)\end{array}$ & $P$-value \\
\hline 1-vessel disease: & 95 (44\%) & $44(48 \%)$ & 0.345 \\
\hline LAD stenosis & $42(20 \%)$ & $20(22 \%)$ & 0.43 \\
\hline LCX stenosis & $25(12 \%)$ & $14(15 \%)$ & 0.241 \\
\hline RCA stenosis & $28(13 \%)$ & $10(11 \%)$ & 0.52 \\
\hline 2-vessel disease: & $74(35 \%)$ & $26(28 \%)$ & 0.652 \\
\hline LAD and LCX stenosis & $32(15 \%)$ & $12(13 \%)$ & 0.543 \\
\hline LAD and RCA stenosis & $19(9 \%)$ & $8(9 \%)$ & 0.234 \\
\hline LCX and RCA stenosis & $23(11 \%)$ & $6(7 \%)$ & 0.341 \\
\hline 3-vessel disease & $45(21 \%)$ & $22(24 \%)$ & 0.712 \\
\hline \multicolumn{4}{|l|}{ Rentrop collateral grades: } \\
\hline 0 & $163(53 \%)$ & & \\
\hline 1 & $51(17 \%)$ & & \\
\hline 2 & & $52(17 \%)$ & \\
\hline 3 & & $40(13 \%)$ & \\
\hline
\end{tabular}

$L A D$ - left anterior descending coronary artery, $L C X$ - left circumflex coronary artery, $R C A$ - right coronary artery. 


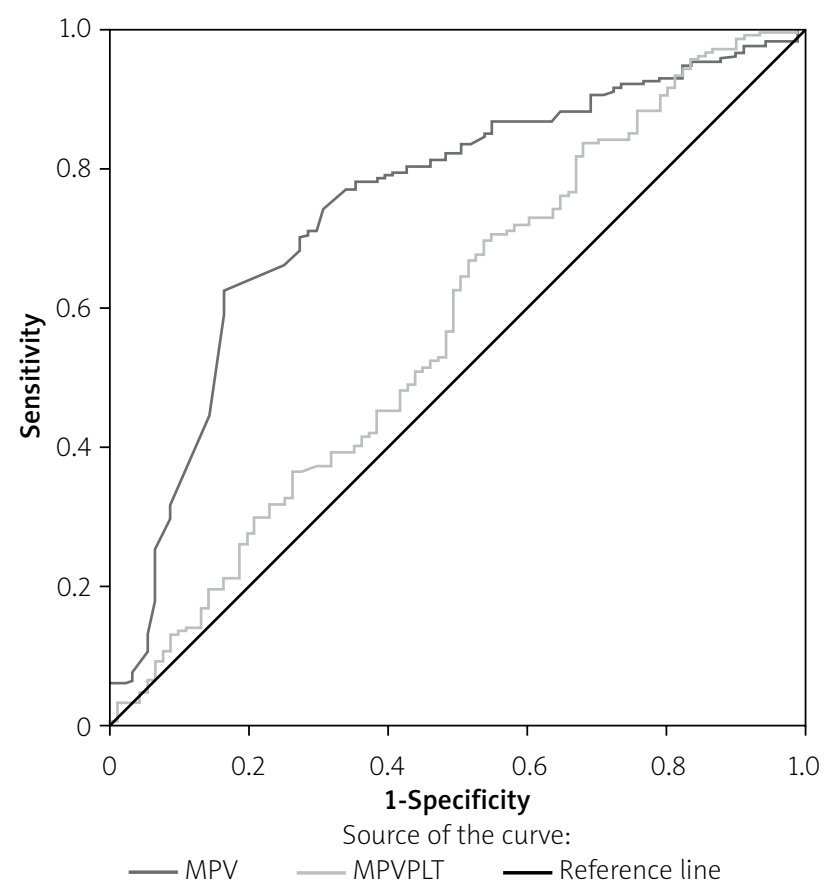

Figure 1. ROC curve analysis of MPV and MPV/P for prediction of inadequate CCD. At the cut-off value of $>9.95 \mathrm{fl}$, sensitivity and specificity of MPV were $71 \%$ and $70 \%$, respectively (AUC $=0.749,95 \% \mathrm{Cl}$ : $0.688-0.810)$. At the cut-off value of $>0.0395 \mathrm{fl} /$ $\left(10^{9} / \mathrm{l}\right)$, sensitivity and specificity of MPV/P were $62.6 \%$ and $51 \%$, respectively $(\mathrm{AUC}=0.576,95 \% \mathrm{Cl}$ : 0.503-0.649)

$A \cup C$ - area under the curve, $\mathrm{Cl}$ - confidence interval.

size rather than platelet count has been found to be associated with platelet function and activation [28]. Accordingly, increased MPV levels were reported to be associated with prethrombotic state in acute coronary syndromes and adverse cardiovascular events [7, 10, $29,30]$. Compared with patients having stable angina and/or non-cardiac pain, patients with the acute coronary syndrome had higher MPV and a lower platelet count [31]. Furthermore, a high MPR has been associated with myocardial infarction, anemia and hepatocellular carcinoma [12-15].

In accordance with our findings, higher MPV levels have been associated with inadequate CCD, and MPV was an independent predictor of degree of $\operatorname{CCD}[2,8$, $9,18]$. In contrast, Sahin et al. found no association between MPV and CCD [2]. They demonstrated that inadequate CCD was independently associated with RDW, but not with other hematological parameters including MPV in patients with stable CAD [2]. However, unlike them we found MPV to be positively and RDW to be negatively associated with inadequate CCD.

The exact mechanism of the association of poor CCD with high MPV levels is unclear. Apart from the primary role of platelet functions in atherosclerosis, inflammation

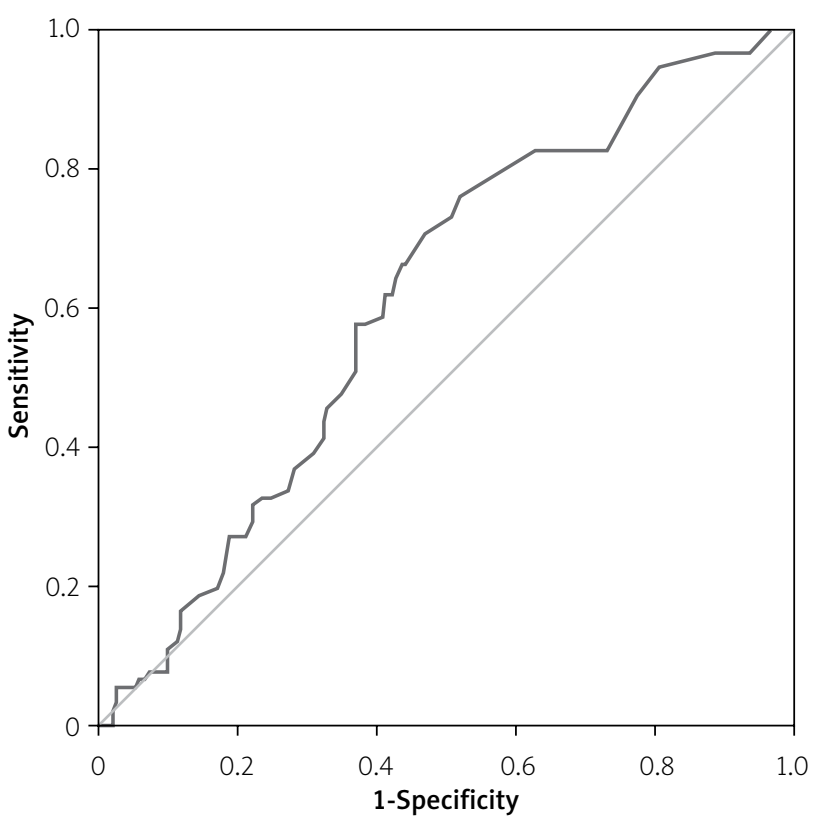

Figure 2. ROC curve analysis of RDW for prediction of adequate CCD. At the cut-off value of $>14.3 \%$, sensitivity and specificity of RDW were $71 \%$ and $53 \%$, respectively (AUC $=0.615,95 \% \mathrm{Cl}: 0.549-$ $0.680)$

AUC - area under the curve, $\mathrm{Cl}$ - confidence interval.

appears to be a probable link between hematological factors and atherosclerosis $[32,33]$. Also there is an interaction between inflammation and new blood vessel formation. Hence, inflammation has been associated with variability of coronary collateralization [23]. Apart from their effects on thrombosis, platelets are also inflammatory cells. They release several substances, including thromboxane A2, platelet factor 4, P-selectin, platelet derived growth factor, and chemotactic and mitogenic factor contributing to vascular neointimal proliferation [34-36]. Large platelets are metabolically and enzymatically more active than small ones [34].

Higher total and LDL cholesterol levels in the inadequate CCD group compared to the adequate CCD group are not surprising since higher cholesterol levels may prevent new vessel development in this population.

Several reports have shown that RDW levels are related to cardiovascular diseases [37]. Red cell distribution width reflects variability in the size of circulating erythrocytes. New young RBC production during anemia results in increased RDW. However, besides anemia, RDW may also be affected by other conditions including inflammatory diseases, iron deficiency, hemolysis and renal failure [37] and also cardiovascular diseases [38, 39]. There have been contradictory results about the association of RDW and CCD $[2,40]$. Sahin et al. reported that RDW was significantly higher in the inadequate CCD group compared to the adequate CCD group [2], whereas other authors did not find any significant association between inade- 
quate CCD and RDW in their study [8]. Our results are inconsistent with them in that we found lower RDW levels in the inadequate CCD group. Therefore, contradictory results in different studies raise a doubt about the association of RDW with CCD in stable CAD patients. Although MPV and RDW have been studied in coronary artery disease previously, the inverse results of RDW are a novel finding of the present study.

In our study, smoking prevalence was significantly higher in the adequate CCD group than the inadequate CCD group. The hypoxic state caused by smoking may induce development of CCD. It has been reported that compared to non-smokers CCD was better among smokers in non-ST elevation MI patients [41]. This effect may cause confounding results as in our study.

\section{Limitations}

This is a retrospective, cross-sectional single-center study. The time of total/subtotal occlusion of the main coronary artery mandating collateral development and following clinical and laboratory changes are unknown. As in previous studies manual platelet counting is not present. Automated cell count techniques may miscount protein particles and fragmented leukocytes as small platelets [42]. Other causes that may alter RDW level such as ferritin, iron, vitamin $B_{12}$ and folate levels were not measured. Lack of measurement of known inflammatory markers such as C-reactive protein and erythrocyte sedimentation rate is another limitation. Another limitation of the present study could be that erythrocyte and platelet indices are studied in many cardiac conditions; thus, significant differences between study and control groups usually lack causative mechanisms. Since it was a retrospective study and there is a lack of strategies aimed at stimulating development of collateral circulation and modest diagnostic accuracies of MPV and RDW, clinical relevance of the study is limited.

\section{Conclusions}

The present study suggests that MPV and MPR may be associated with degree of collateral development in chronic stable CAD. However, the negative association of RDW with inadequate CCD, in combination with previous contradictory reports, raises a doubt about the possible value of RDW in stable CAD. Although these parameters may be affected by various conditions, a high MPV may lead clinicians to suspect possible inadequate collateral development in stable CAD patients.

\section{Conflict of interest}

The authors declare no conflict of interest.

\section{References}

1. Çelik T, Çelik M, Iyisoy A. Coronary collateral circulation. Turk J Cardiol 2010; 38: 505-14.
2. Sahin I, Karabulut A, Kaya A, et al. Increased level of red cell distribution width is associated with poor coronary collateral circulation in patients with stable coronary artery disease. Turk J Cardiol 2015; 43: 123-30.

3. Demirbag R, Gur M, Yilmaz R, et al. Influence of oxidative stress on the development of collateral circulation in total coronary occlusions. Int J Cardiol 2007; 116: 14-9.

4. Tsiara S, Elisaf M, Jagroop IA, et al. Platelets as predictors of vascular risk: is there a practical index of platelet activity? Clin Appl Thromb Hemost 2003; 9: 177-90.

5. Davì G, Patrono C. Platelet activation and atherothrombosis. New Eng J Med 2007; 357: 2482-94.

6. Park Y, Schoene N, Harris W. Mean platelet volume as an indicator of platelet activation: methodological issues. Platelets 2002; 13: 301-6.

7. Yilmaz MB, Cihan G, Guray Y, et al. Role of mean platelet volume in triagging acute coronary syndromes. J Thromb Thrombolysis 2008; 26: 49-54.

8. Ege MR, Acıkgoz S, Zorlu A, et al. Mean platelet volume: an important predictor of coronary collateral development. Platelets 2013; 24: 200-4.

9. Ornek E, Kurtul A. Relationship of mean platelet volume to lymphocyte ratio and coronary collateral circulation in patients with stable angina pectoris. Coron Artery Dis 2017; 28: 492-7.

10. Khandekar M, Khurana A, Deshmukh S, et al. Platelet volume indices in patients with coronary artery disease and acute myocardial infarction: an Indian scenario. J Clin Pathol 2006; 59: 146-9.

11. Pekdemir H, Polat G, Cin VG, et al. Elevated plasma endothelin-1 levels in coronary sinus during rapid right atrial pacing in patients with slow coronary flow. Int J Cardiol 2004; 97: 35-41.

12. Cure MC, Cure E, Kirbas A, et al. The effects of Gilbert's syndrome on the mean platelet volume and other hematological parameters. Blood Coagul Fibrinolysis 2013; 24: 484-8.

13. Ju HY, Kim JK, Hur SM, et al. Could mean platelet volume be a promising biomarker of progression of chronic kidney disease? Platelets 2015; 26: 143-7.

14. Cure E, Balik MS, Cumhur Cure M, et al. Is the mean platelet volume predictive of hip fractures in the elderly? Ann Lab Med 2013; 33: 367-70.

15. Azab B, Torbey E, Singh J, et al. Mean platelet volume/platelet count ratio as a predictor of long-term mortality after non-ST-elevation myocardial infarction. Platelets 2011; 22: 557-66.

16. Rentrop KP, Thornton JC, Feit F, et al. Determinants and protective potential of coronary arterial collaterals as assessed by an angioplasty model. Am J Cardiol 1988; 61: 677-84.

17. Habib G, Heibig J, Forman S, et al. Influence of coronary collateral vessels on myocardial infarct size in humans. Results of phase I thrombolysis in myocardial infarction (TIMI) trial. The TIMI Investigators. Circulation 1991; 83: 739-46.

18. Meier P, Gloekler S, Zbinden R, et al. Beneficial effect of recruitable collaterals: a 10-year follow-up study in patients with stable coronary artery disease undergoing quantitative collateral measurements. Circulation 2007; 116: 975-83.

19. Werner GS, Ferrari M, Heinke S, et al. Angiographic assessment of collateral connections in comparison with invasively determined collateral function in chronic coronary occlusions. Circulation 2003; 107: 1972-7.

20. Imhof BA, Aurrand-Lions M. Angiogenesis and inflammation face off. Nature Med 2006; 12: 171-2. 
21. Gok M, Kundi H, Kiziltunc E, et al. Endocan levels and coronary collateral circulation in stable angina pectoris: a pilot study. Angiology 2018; 69: 43-8.

22. Açar G, Kalkan ME, Avci A, et al. The relation of platelet-lymphocyte ratio and coronary collateral circulation in patients with stable angina pectoris and chronic total occlusion. Clin Appl Thromb Hemost 2015; 21: 462-8.

23. Kurtul A, Yarlioglues M, Murat SN, et al. Usefulness of the platelet-to-lymphocyte ratio in predicting angiographic reflow after primary percutaneous coronary intervention in patients with acute ST-segment elevation myocardial infarction. Am J Cardiol 2014; 114: 342-7.

24. Hotchkiss RS, Karl IE. The pathophysiology and treatment of sepsis. N Eng J Med 2003; 348: 138-50.

25. Duffy BK, Gurm HS, Rajagopal V, et al. Usefulness of an elevated neutrophil to lymphocyte ratio in predicting long-term mortality after percutaneous coronary intervention. Am J Cardiol 2006; 97: 993-6.

26. Braekkan S, Mathiesen E, Njølstad I, et al. Mean platelet volume is a risk factor for venous thromboembolism: the Troms $\varnothing$ study. J Thromb Haemost 2010; 8: 157-62.

27. Chung I, Choudhury A, Lip GY. Platelet activation in acute, decompensated congestive heart failure. Thrombosis Res 2007; 120: 709-13.

28. Thompson CB, Eaton KA, Princiotta SM, et al. Size dependent platelet subpopulations: relationship of platelet volume to ultrastructure, enzymatic activity, and function. Br J Haematol 1982; 50: 509-19.

29. Bulur S, Önder HI, Aslantas Y, et al. Relation between indices of end-organ damage and mean platelet volume in hypertensive patients. Blood Coagul Fibrinolysis 2012; 23: 367-9.

30. Ranjith M, Divya R, Mehta V, et al. Significance of platelet volume indices and platelet count in ischaemic heart disease. J Clin Pathol 2009; 62: 830-3.

31. Tanboga IH, Topcu S, Nacar T, et al. Relation of coronary collateral circulation with red cell distribution width in patients with non-ST elevation myocardial infarction. Clin Appl Thromb Hemost 2014; 20: 411-5.

32. Perlstein TS, Weuve J, Pfeffer MA, et al. Red blood cell distribution width and mortality risk in a community-based prospective cohort. Arch Intern Med 2009; 169: 588-94.

33. Martin J, Shaw T, Heggie J, et al. Measurement of the density of human platelets and its relationship to volume. $\mathrm{Br} J$ Haematol 1983; 54: 337-52.

34. Kamath S, Blann A, Lip G. Platelet activation: assessment and quantification. Eur Heart J 2001; 22: 1561-71.

35. Corash L, Tan H, Gralnick HR. Heterogeneity of human whole blood platelet subpopulations. I. Relationship between buoyant density, cell volume, and ultrastructure. Blood 1977; 49: 71-87.

36. Ghaffari S, Pourafkari L, Javadzadegan $\mathrm{H}$, et al. Mean platelet volume is a predictor of ST resolution following thrombolysis in acute ST elevation myocardial infarction. Thrombosis Res 2015 ; 136: 101-6.

37. Wen Y. High red blood cell distribution width is closely associated with risk of carotid artery atherosclerosis in patients with hypertension. Exp Clin Cardiol 2010; 15: 37-40.

38. Förhécz Z, Gombos T, Borgulya G, et al. Red cell distribution width in heart failure: prediction of clinical events and relationship with markers of ineffective erythropoiesis, inflammation, renal function, and nutritional state. Am Heart J 2009; 158: 659-66.
39. Felker GM, Allen LA, Pocock SJ, et al. Red cell distribution width as a novel prognostic marker in heart failure: data from the CHARM Program and the Duke Databank. J Am Coll Cardiol 2007; 50: 40-7.

40. Tonelli M, Sacks F, Arnold M, et al. Relation between red blood cell distribution width and cardiovascular event rate in people with coronary disease. Circulation 2008; 117: 163-8.

41. Sen HS, Abakay O, Tanrikulu AC, et al. Is a complete blood cell count useful in determining the prognosis of pulmonary embolism? Wien Klin Wochenschr 2014; 126: 347-54.

42. Li S, Salhany KE. Spurious elevation of automated platelet counts in secondary acute monocytic leukemia associated with tumor lysis syndrome. Arch Pathol Laboratory Med 1999; 123: 1111-4. 